\title{
Origen y andanzas del término económico dita*
}

\author{
Origin and vicissitudes of the business term dita
}

\author{
Franz Rainer \\ WU Viena \\ franz.rainer@wu.ac.at \\ ORCID ID: https://orcid.org/0000-0001-7258-535X
}

RESUMEN: La etimología del término económico dita, que sobrevive en América y en Andalucía, es considerada todavía como "discutida" en la versión en línea del DRAE. Cuervo, en su tiempo, propuso un origen italiano, mientras Corominas dio la preferencia a un origen catalán. En el presente artículo me propongo mostrar que el término tuvo efectivamente un origen italiano, pero fue transmitido al español a través del catalán. Se mostrará, además, que las hipótesis de Cuervo y Corominas sobre las palabras concretas que habrían servido de modelo al español son incorrectas. El étimo está representado más bien por el italiano detta, que designaba inicialmente en la Edad Media el enunciado del banquero que acompañaba el giro bancario de una cuenta a otra.

Palabras clave: dita, lenguaje económico, etimología, préstamo, catalanismo, italianismo.

ABSTRACT: The etymology of the business term dita, which survives in America and in Andalusia, is still considered unresolved in the online version of the dictionary of the Real Academia. Over a hundred years ago, Cuervo proposed an Italian origin, while Corominas later preferred a Catalan origin. In this article, I intend to show that the term indeed has Italian roots, but entered Spanish via Catalan. I will also argue that the hypotheses put forward by Cuervo and Corominas concerning the concrete word that served as a model were incorrect. The correct etymon is Italian detta, which in the Middle Ages referred to the words pronounced by a banker when transferring money from one account to another.

Keywords: dita, business language, etymology, loan word, Catalanism, Italianism.

* Quisiera dar las gracias a un anónimo evaluador por haberme llamado la atención sobre la versión electrónica del Fichero General de la Real Academia, cuyas fichas me han permitido adelantar la fecha de primera documentación de dita en español 12 años y completar la información sobre los usos en los dialectos de España y de América, así como a Pilar Pérez Cañizares por la atenta lectura del texto final. 


\section{USO ACTUAL Y PASADO DEL TÉRMINO EN ESPAÑOL}

Para el hablante medio del español estándar de España dita es, hoy por hoy, un término que ya no pertenece a su vocabulario activo, y probablemente tampoco pasivo. Sobrevive, sin embargo, en variedades regionales de España y de América. El DRAE lo documenta con el significado 'deuda' en varios países latinoamericanos (Chile, Guatemala y México), así como en Albacete (España). Calero López de Ayala (1987) también lo ha hallado en la provincia de Cuenca (España). En Andalucía, según el $D R A E$, se utiliza con el significado 'pago a plazos', mientras el significado 'persona o efecto que se señala como garantía de pago’ ya habría caído en desuso.

dita $^{1}$

Etim. disc.; $c f$. it. ant. ditta 'cosas dichas', detta 'cosas debidas' y cat. dita. 1. f. Alb., Chile, Guat. y Méx. deuda (obligación de pagar).

2. f. And. Pago a plazos, en pequeñas cantidades, fijadas por el comerciante o por el cliente $y$, en ocasiones, con incremento del interés sin el conocimiento de este.

3. f. desus. Persona o efecto que se señala como garantía de un pago.

La información del $D R A E$ sobre el uso de la acepción 'deuda' en América es avalada por los diccionarios especializados. Morínigo (1985) lo trae como usual en América Central, Chile y México, países a los cuales el reciente Diccionario de americanismos de la Asociación de Academias de la Lengua Española (2010) añade el Perú1. López Blanquet (1992) lo documenta en Uruguay. La acepción andaluza 'pago a plazos' es la única que recoge el $D E A$, ilustrándola con dos ejemplos del escritor sevillano Alfonso Grosso (1928-1995). La definición del diccionario, es decir "préstamo a elevado interés, pagadero por días con el capital", está sacada del Vocabulario andaluz de Alcalá Venceslada (1951), que proporciona una cita del padre Luis Coloma (Jérez de la Frontera, 1851-1915). El Tesoro Léxico de las Hablas Andaluzas (Alvar Ezquerra, 2000) también retoma la definición de Alcalá Venceslada, y añade dos acepciones más: "2 ¿Deuda?, crédito. [VAF]. 3 Compra a plazos. [HJ: Jerez de la Frontera (Ca.)]”. Alcalá Venceslada señala, además, la existencia, en el dialecto andaluz, de dos derivados de dita: ditera 'fiadora, mujer que vende al fiado, a dita' y ditero, ra 'persona que presta a dita'.

La tercera acepción del DRAE, marcada como desusada, corresponde al único significado que recoge el Diccionario de Autoridades (Real Academia

\footnotetext{
${ }^{1}$ En cuanto a la etimología, este diccionario comete un error, declarando dita de origen indiano antillano. Esta atribución, por supuesto, solo es correcta para el uso especial que se hace de dita en Puerto Rico, donde se refiere a una 'vasija hecha con la mitad de la corteza de una higue$\mathrm{ra}$, de un coco o de una calabaza'. Este uso nada tiene que ver con el uso económico que nos ocupa en el presente trabajo.
} 
Española 2002 [1732]): “DITA. s. f. Persona o efecto que se señala para pagar lo que se debe, ò para assegurar la satisfacción de lo que se compra ò toma prestado. Lat. Nomen, inis. Certum aes alienum". El ejemplo más antiguo de este diccionario proviene de la segunda parte del Guzmán de Alfarache de Mateo Alemán (1604): "Quando haviamos de dar una partida, reconocíamos la dita, y siendo persóna de quien sabíamos que tenía de que pagar... se la dábamos llanamente, aunque algunas veces aconteció faltarnos de estas ditas algunas que teníamos por las mejóres". El primer ejemplo que proporciona el NTLE es de poco anterior. Francisco de Rosal, en 1601, explicó: “dita, para pagar, es dicta, que quiere decir nombrada y señalada, y nace de la fórmula lat. dicere diem, que es señalar plazo y día para pagar". La mención siguiente del NTLE, que proviene del diccionario español-francés (1604) de Ioan Palet, es de interés porque da como equivalente francés simplemente dette, es decir, 'deuda'.

Nuestro término, como acabamos de ver, se documenta en la lexicografía a partir de inicios del siglo XVII, pero en los textos ya aparece en el siglo precedente. El primer ejemplo que he podido hallar proviene del Fichero General de la Real Academia ${ }^{2}$. Se encuentra en una fuente cubana fechada en 1520: "Todo lo quería aver dado a tales preçios e tales ditas, y dígolo por desempalagarme de tanto hato". La interpretación más probable de la palabra en este contexto es 'deudor'. Los ejemplos siguientes se encuentran en la traducción de un dictamen de 1532 de los teólogos de la Sorbona sobre algunos casos de conciencia que les habían sometido los mercaderes de la nación española de Amberes $^{3}$. La palabra aparece por primera vez en el segundo caso:

Algunas vezes aconteçe que los mercaderes no quierente ner $[i . e$. quieren tener] que hazer con los pueblos y dizen al fiançero "Señor, nos ottos no queremos tener que hazer con los pueblos, pero sy vos nos dais buena dita abonada que nos responda destos dineros dentro del año somos contentos de dar luego los dineros y donde el emperador es contento de dar quinze por çiento nos contentamos que no pierda sino treze". Va el fiançero a algunos hombres ricos que tienen credito y conçierta se con ellos y da les los [sic] obligaçiones de los pueblos los quales mercaderes de credito responden el dinero dentro de un año a los mercaderes que lo dan luego y por aquella responsion que hazen de pagar dia adiado puesto que los pueblos no pagassen y ellos sin poner otta cosa de su casa, llevan aquellos doss por çiento de los quinze que el emperador pierde y algunas vezes mas y otras menos. Preguntase sy es liçito (Goris, 1925: 526-527).

En la situación descrita, los mercaderes no quieren correr el alto riesgo de un préstamo directo al rey y exigen al intermediario que encuentre una "buena

\footnotetext{
${ }^{2}$ Consultable en línea: <http://www.rae.es/recursos/banco-de-datos/fichero-general >.

${ }^{3}$ El texto se conserva en el Codex hispanicus, n. ${ }^{\circ}$ 30, de la Biblioteca de Múnich. Ha sido editado por Goris (1925: 510-545) y por Beltrán de Heredia (1935: 517-528). Me baso aquí en la edición de Goris, que transcribo tal cual.
} 
dita abonada", es decir un avalista que, a cambio de una comisión del dos por ciento, les pague en caso de que no lo hiciera el rey. Los avalistas, como se nos dice, tenían que ser "hombres ricos que tienen crédito" o "mercaderes de crédito", expresiones que podrían sustituirse en el contexto dado a la expresión "buena dita abonada" sin alterar el sentido.

En el noveno caso (Goris, 1925: 531), dita aparece dos veces, primero con el significado 'mercader de crédito' y luego en la locución correr la dita, que habrá que interpretar como 'correr el riesgo':

Diego, mercader, querria hazer una fiança por que tiene neçessidad de dinero y por esto querria comprar de Alonso çiertas [sic] sacos de pimenda al fiado, pero no tiene credito con el, va se a Hernando que es buena dita en la bolsa y dize "Señor yo querrio $[s i c]$ çien sacos de pimenda fiados por un ano [sic]". Conçertase del preçio con el, va Hernando por el credito que tiene y toma fiados por un ano los çien sacos de pimenda de Alonso y vende la fiada a Diego por el mismo tiempo a quatro o çinco por çiento sin poner nada de su casa, gaña aquello solo por que corre la dita de pagar a Alonso dia adiado aunque Diego no pagasse a el. Preguntase etc.

No he encontrado en toda la documentación que he manejado otro ejemplo de la locución correr la dita, por lo cual me inclino a la hipótesis más cautelosa de que se trata de una elipsis ocasional de la secuencia más explícita que encontramos en el siguiente ejemplo, sacado de un dictamen de Francisco de Vitoria (Goris, 1925: 533):

Antonio esta en feria de Mayo y tiene necessidad dee mill ducados de presente los quales tiene en Sevilla en duebdas o en ottas manera para en fin de setiembre querria socorrerse dellos en la dicha feria de mayo. Da se los Patriçio aunque a Patriçio no yncurre de los yr a cobrar a Sevilla ny tiene para que los tener alla. Da le Antonio çedulas paraque le sean pagados en Sevilla para en fin de dicho mes de setiembre y por mill ducados que aquí reçibe paga en Sevilla mill y veinte o mill y veinte y çinco [...]. Reçibe Patricio ultra sortem partim para costas de ferias, y responsiones, y corretajes, $\mathrm{y}$ portos de cartas, y partim por su trabajo y yndustria y de su fator de Sevilla partim por el riesgo que corre de la dita de Antonio, partim por [...].

En este ejemplo Patricio, que ha dado a Antonio mil ducados en efectivo en la feria de mayo de Medina del Campo a cambio de una letra sobre un deudor que Antonio tiene en Sevilla, corre el riesgo de la dita de Antonio hasta el momento en que cobre la letra. La interpretación más plausible de dita en este contexto parece ser la de 'deuda'. Más remota me parece la posibilidad de que dita se refiera aquí al deudor de Antonio en Sevilla, porque en caso de que aquel no pagase, Antonio mismo tendría que honorar la letra protestada. Así que el riesgo fundamental es el de la solvencia de Antonio. Otro ejemplo idéntico se encuentra un poco más adelante en el segundo caso tratado por Vitoria (Goris, 1925: 537). 
Como se ve, dita se nos presenta como palabra polisémica desde los primeros ejemplos documentados. Puede referirse tanto a la deuda misma, como también a una persona (deudor, avalista). Estos conceptos tienen lazos metonímicos evidentes entre sí. Ambos significados se documentan ampliamente a partir de mediados del siglo XVI, tanto en España como en América. Para México, se puede citar un ejemplo temprano con una clara referencia personal, sacado de una carta del 1 de enero de 1568: "Tengo una dita en la cárcel que me debe 4.800 pesos y dice que si no lo suelto que no hay cobrar de él" (Carande, 1978: 191). El único ejemplo que contiene el Léxico Histórico del Español de México (Company y Melis, 2002), sin embargo, concierne el significado 'deuda': "el susso dicho se hazía prenda de algunos bienes de dichos yndios, naturales de dicha jurisdicion, para asegurar sus ditas" (1694).

Los ejemplos del CORDE pueden también interpretarse en uno u otro sentido. 'Deuda': "Avn no me acordaua dessa dita, que cincuenta semejantes cobraría hombre si procurase de poner diligencia" (1536, Gaspar Gómez de Toledo, Tercera parte de la tragicomedia de Celestina); 'deudor': "el pobre compra más caro porque temen que no es buena dita" (a 1540, Francisco de Osuna, Quinta parte del Abecedario espiritual). Además, hay en el CORDE un ejemplo, debido al mismo autor, donde dita parece referirse al crédito personal, a la reputación de un mercader:

Ninguna ave puede caçar buena pieça quando el águila sale a caçar, porque todo lo apaña ella y no dexa sino las cosas viles que tienen poca carne. De esta manera haze el rico mercader con los mercaderes pobres, que no les da lugar, ni les da parte sino de las cosas donde ay poca o ninguna ganancia. É, con su buena dita, se lo quiere ganar todo y escoger los mejores bocados, [...] (a 1540, Quinta parte del Abecedario espiritual, p. 590).

En este ejemplo, ni *con su buena deuda ni *con su buen deudor serían alternativas viables. Como no he encontrado otros ejemplos con este significado, conviene quizás interpretar este uso como extensión $-\mathrm{o}$, mejor dicho, reducción- ocasional de la expresión buena dita en su significado corriente 'mercader de gran crédito'.

Los dos significados principales, 'deuda' y 'deudor', los documenta también Gonzalo de Correas: "Mala dita. Del mal pagador. Su kontrario es "buena dita”. [...] Dita perdida. Son ditas perdidas. Las ke no ai kómo kobrarlas" (1627, Vocabulario de refranes y frases proverbiales). Pero después de Correas, los ejemplos empiezan a escasear en el CORDE. Lo que no significa que no se puedan encontrar, de vez en cuando, ejemplos incluso mucho más tarde, como el siguiente de inicios del siglo XIX que proporciona la Hemeroteca Digital de la Biblioteca Nacional de España: "este debia á Mr. Fualdés [...] 15 francos de la venta de la hacienda de Flars, con mas 150 francos de otras ditas" (Mercurio de España, 11/1817, p. 35). 
La acepción andaluza 'pago a plazos' mencionada al inicio la encuentro por primera vez en el CORDE en una carta de 1777 de Jovellanos a Campomanes que alude explícitamente a la situación en Sevilla:

En Sevilla, por ejemplo, todo el pueblo compra al fiado, y a pagar a ditas. Esto quiere decir que compra a precios altísimos, ya porque en estas ventas no hay regateo y la boca del mercader es la regla del precio, y ya porque es necesario, y aún justo, que el valor del género vendido se recargue el interés correspondiente a los plazos señalados para la paga. En esto siente el pueblo un considerable perjuicio, que influye sensiblemente en la alteración de los jornales y del precio de las obras de industria. Un monte pío cortaría de raíz este inconveniente.

El significado '(pago a) plazos' es otro caso de extensión metonímica, sin duda a partir del significado 'deuda'.

\section{LAS HiPÓTESIS ETIMOLÓGICAS DE CUERVO Y COROMINAS}

Ya hemos visto arriba que Francisco de Rosal, en el primer diccionario etimológico de la lengua española, relacionaba dita con la forma femenina del adjetivo dito 'dicho', que en determinadas variedades del español se usaba en expresiones como la dita ciudad y otras del mismo tipo. El primer acercamiento serio al problema lo debemos a Rufino José Cuervo, quien en 1909 dedicó al término una de sus "antiguallas del habla hispano-americana". Había identificado correctamente dita como arcaísmo latinoamericano. En cuanto al origen del término, constata: "A todas luces nos vino de Italia, aunque en este punto se me ofrecen dificultades" (Cuervo, 1909: 287). Estas dificultades consistían en que pensaba que los usos de detta descritos en el diccionario de la Accademia della Crusca (1612) eran atribuibles a dos étimos latinos diferentes, DICTA '(lo) dicho' (por ejemplo en la locución a detta di $X$ 'según lo que $\mathrm{X}$ dice') y DEBITA 'deudas', que Cuervo veía detrás de usos como le dette sono cresciute per l'usura 'las deudas aumentaron por la usura' o è una buona/cattiva detta 'es un buen/mal pagador'. Estas últimas locuciones corresponden perfectamente al buena/mala dita del español del Siglo de Oro, pero Cuervo veía un escollo formal insalvable. Para explicar la calidad vocálica de dita, pensaba, había que tomar como punto de partida no el toscano detta sino ditta (o dita), variante que efectivamente existe en las variedades septentrionales del italiano. Ahora bien, el septentrional dita correspondía al detta toscano solo con el significado '(lo) dicho', pero no con el significado 'deuda' que Cuervo pensaba que estaba en la base de las locuciones buona/cattiva detta (con extensión semántica de 'deuda' a 'deudor'). Incapaz de resolver esta aparente contradicción entre forma y contenido, Cuervo barruntó que los italianos hubieran confundido el DICTA 
con el DEBITA, confusión que los españoles que conocieron el término en Italia habrían trasvasado a España: "Los españoles que oyeron en Italia la palabra, la interpretaron vagamente, y con la misma vaguedad la usaron los que la oyeron ya en España, conforme vamos á verlo" (Cuervo, 1909: 289).

El erudito colombiano acertó en atribuir al término un origen italiano, pero se equivocó tanto en la etimología del italiano detta/ditta como en el canal de transmisión. La rica documentación del Siglo de Oro español que presenta adolece de su origen exclusivamente literario, que no permitía a Cuervo delinear con precisión el abanico semántico del término, ni entrever el canal de transmisión. El primer significado que Cuervo identificó era el de 'crédito comercial de alguno, solvencia' (Cuervo, 1909: 289), pero aunque también he aducido arriba un único ejemplo con este significado, los que aduce Cuervo me parecen todos compatibles con los dos significados fundamentales 'deuda' y 'deudor'. Prosigue Cuervo (1909: 290) atribuyendo a dita el significado 'pagaré', supuestamente una extensión del significado 'deuda' a través de 'promesa de pago'. Pero otra vez sus ejemplos no avalan este significado, siendo compatibles con lecturas más conservadoras. Por ejemplo, en los versos "Ayer puso en sus ditas todas cobro, Mas hoy ya torna al logro", ditas puede sustituirse sin problemas con deudas, y no he visto ningún ejemplo donde el significado más concreto de 'pagaré' fuera necesario. Lo mismo se puede decir de los significados 'letra de cambio' ${ }^{4}$ y 'partida de una cuenta' que Cuervo (1909: 291-292) atribuye a dita. Una deuda puede materializarse de muchas maneras, como saldo negativo de una cuenta, como efecto comercial, o simplemente a través de la palabra dada, pero eso no quiere decir que deuda signifique 'saldo negativo, 'pagaré', 'letra de cambio', o 'promesa de pago'. Para atribuir a dita estos significados más específicos, se necesitarían contextos menos ambiguos que los aducidos por Cuervo.

Corominas, en el DCECH (II 432a), tampoco se dejó convencer por la explicación de Cuervo y propuso a su vez un origen catalán ${ }^{5}$ :

en vista de que el vocablo aparece en el aragonés de Segorbe con el sentido 'lo que se ofrece cuando se subasta algo' (Torres Fornés), lo cual coincide con el significado de dita en catalán [1422, Alcover, Fabra, Escrig; S. XIII en otras acs.], y en vista de que ditta es forma meramente local en italiano, es preferible derivar del cat. dita.

Como vamos a ver en la sección 3, Corominas se equivocó al rechazar la hipótesis de Cuervo de un origen italiano, por lo menos como etimología remo-

\footnotetext{
${ }^{4}$ La adscripción a dita del significado 'letra de cambio' puede haber sido influenciada por la entrada dita en el diccionario de Oudin (1616): "Dita, dita buena o mala, bonne ou mauvaise partie. Ce mot vient de dire et bailler parole d'estre solvable: et selon aucuns c'est une lettre de change".

${ }^{5}$ Terlingen (1943) en su clásica monografía tampoco incluyó dita entre los italianismos.
} 
ta, aunque también tenía una parte de razón, porque el término efectivamente transitó por el catalán. El uso catalán pertinente, sin embargo, no fue el de las subastas al cual alude Corominas y que falta en español, sino un uso estrechamente técnico del mundillo bancario medieval, familiar a los historiadores de la banca, pero que falta en los diccionarios compilados por académicos o filólogos y por eso se escapó a su atención.

\section{DETTA/DITA, TÉRMINO BANCARIO MEDIEVAL}

Uno de los mayores rompecabezas del comercio medieval era el de la transferencia de dinero. Transportar grandes sumas de metálico de un lugar a otro no constituía solo un problema práctico, sino también de seguridad. Por eso, muy pronto los mercaderes idearon métodos que permitían pagar deudas sin transportar dinero. En el comercio internacional, el instrumento desarrollado a tal efecto fue la letra de cambio, que en la Edad Media todavía requería una diferencia de lugar y por ende de moneda (de ahí su nombre), mientras en el comercio local el método consistía en el giro bancario de una cuenta a otra. En vez de entregar al otro una bolsa llena de monedas, las partes de la transacción acudían a su banquero común al cual el deudor daba la orden de transferir la suma debida de su propia cuenta a la del otro. Era esta operación de giro bancario que se llamaba, en el florentino antiguo, detta. La operación se llamaba así porque en los primeros tiempos requería la presencia en el acto de las tres partes mencionadas, los titulares de las cuentas y el banquero, y porque la orden de giro se daba oralmente. Melis (1962: 425) describe así la operación:

L'operazione richiedeva è noto la presenza dei due correntisti presso il comune banchiere: al quale il "girante" "diceva" il suo intendimento e quegli, a sua volta, "diceva" di consentire a favore del "giratario". La vera e propria detta era quella che pronunciava il banchiere, cui spettava, infatti, la decisione della voltura del credito.

Añade Melis en una nota que en los libros del banquero, "il soggetto del verbo dire è sempre il banchiere". La detta que contaba jurídicamente era la del banquero, quien con su enunciado manifestaba que estaba de acuerdo en transferir la suma de una cuenta a la otra y quien también corría el riesgo de crédito en caso de que la suma transferida proviniera de un crédito del banquero al girador. La descripción de la operación por parte de Melis se basa en un "quaderno delle dette", es decir, un libro de giros bancarios, de 1399 enviado a Prato desde Barcelona, que se ha conservado en el archivo Datini de Prato. Las entradas tienen todas la siguiente forma (Melis, 1962: 426): 
A Franciescho Safabricha, in Giame Puedilucho

lb. 301 s. 5

Da Giame Bibero, drapieri, per canbî di Brugia, in Antonio Rapustiero lb. 221 s. 2 d. 8

Estas entradas, hay que leerlas de la manera siguiente: Ha sido transferida a la cuenta de Franciescho Safabricha en el banco de Giame Puedilucho la suma de 301 libras y 5 sueldos. / Ha sido transferida a nuestra cuenta por parte de Giame Bibero... en el banco de Antonio Rapustiero la suma de 221 libras, 2 sueldos y 8 denarios. El banquero se señala con la preposición in, el giratario con $a$, y el girador con $d a$. En las escrituras correspondientes del libro mayor aparece explícitamente el verbo dire 'decir', con el banquero como sujeto':

Franciescho Safabricha dèe dare, a dì primo di febraio, lb. tecientouna s. cinque, dìsolli per noi Giame Puedilucho.

En cuanto a la etimología de detta, pues, no cabe la menor duda de que el término debe relacionarse con dire 'decir'. No comparto, sin embargo, la opinión de Melis (1962: 425), seguido en este punto por Trolli (1998: 20), quien interpeta detta como "participio passato del verbo dire". Ni Melis ni Trolli nos dicen qué sustantivo femenino, en su opinión, subyacería a tal forma elíptica. Me parece más correcto decir que detta remonta al neutro plural latino DICTA 'lo dicho, enunciado', que a su vez sí es un participio sustantivado (latino, no italiano). Tal sustantivo se ha conservado hasta el día de hoy en italiano en la locución a detta di $X$ 'según lo que dice $X$ ', literalmente 'según el enunciado de X'. Su aplicación, en el italiano antiguo, al caso específico del enunciado o acto de habla del banquero en el contexto del giro bancario tenía que ser muy natural en una época cuando el sustantivo gozaba todavía de un uso menos restringido que en la actualidad.

Es importante pararse un momento para analizar más de cerca la etimología del término detta en italiano, porque no solo el artículo de Cuervo sino también los diccionarios histórico-etimológicos del italiano contienen aserciones dudosas a ese respecto. El DELI no trae nuestro término bancario porque ya no es de uso actual, pero en la entrada ditta, palabra que hoy en día significa 'compañía' y que en este sentido correctamente se presenta como forma elíptica del veneciano dita compagnia 'dicha compañía', ese diccionario se equivoca cuando da como primera documentación un ejemplo de Luca Pacioli de 1494 con el significado 'certificato di pagamento, ricevuta, firma', que hay que relacionar más bien con el término ditaldetta que aquí nos ocupa, como bien vio Trolli (1998: 20). El $G D L I$ por su parte pone todos los usos económicos en la entrada $\operatorname{detta}^{3}$, definida como "Ant. Credito", que a través del francés dette se hace remontar al latino

\footnotetext{
${ }^{6}$ Conde y Delgado de Molina (1988: 128), a propósito de documentos catalanes, escribe: "De hecho, la mejor traducción del verbo dir de los asientos bancarios sería "abonar en cuenta de"”.
} 
DEBITA. Ahora bien, a la luz de lo que acabamos de ver, es probable que por lo menos una parte de los ejemplos deban colocarse más bien en la entrada detta ${ }^{1}$, "Il dire, ciò che si dice, le cose dette", que viene del latino DICTA. Un primer candidato potencial es el siguiente ejemplo del siglo XIII: "Ebbi i quali Noffo pagoe per me nel Tenpio di Parigi, di XI di magio ano ottanta otto, i quali m'asengnò Girardo Cialciato di Kiermonte in dette ed in conta[n]ti". El autor de estas líneas recibió de Girardo Cialciato di Kiermonte un pago "in dette ed in contanti”. La lectura 'en giros bancarios y en efectivo' parecería aquí más plausible que 'en deudas y en efectivo'. Si el significado fuera verdaderamente 'en deudas', habría que pensar en una cesión de deudas, por ejemplo mediante una letra de cambio librada sobre un deudor solvente, pero en este supuesto, ¿por qué no escribió simplemente lettere di cambio? El siguiente ejemplo catalán contemporáneo, donde no cabe duda de que se habla de ditas y no de deudas (que en catalán se dicen deutes), es enteramente paralelo al ejemplo italiano y por ende apoya la lectura 'giro bancario' de este: “que los dits P. Vidal e G. Juglar paguen ... decontinent $\mathrm{C}$ lliures co es L lliures de comptans e altres L lliures en dita en dita Taula ${ }^{7}$ de la ciutat" (Usher, 1943: 555). Otro ejemplo que parece aludir directamente a la operación del giro bancario (compárese el uso del verbo voltare con voltura en la cita de Melis arriba) es el siguiente de Francesco d'Ambra (Florencia, 1499-1558), donde detta podría interpretarse como 'partida': "Avea da Bartolo / aver cento ducati ... / E noi pregammolo / sendo venuto 'l tempo che voltasse la / detta a voi". Los ejemplos que contienen las expresiones buona/ cattiva detta 'buen, mal deudor, pagador' (ambas del siglo XVI) deberán considerarse también como evoluciones semánticas sobre la base del significado bancario, como sugiere el paralelismo del catalán y del español. Incluiría también en este grupo el ejemplo siguiente de Annibal Caro (1507-1566), al cual el GDLI reserva una acepción separada ("Patto, oferta"), no documentada en otros textos: "Questa detta m'è parsa onorevole e da farne presto ritratto". Lo interpreto como: Este deudor (o deuda) me pareció honorable y tal de cobrar pronto. Los redactores del LEI tendrán que reanalizar a fondo todo el material del italiano antiguo que contiene detta en un sentido económico, para establecer cuáles significan 'giro bancario' y cuáles 'deuda' y cuáles de los ejemplos con significado 'deuda' contienen extensiones metonímicas del significado 'giro'/‘partida' y cuáles provienen realmente de DEBITA a través del francés dette. Edler (1934: 104) trae un ejemplo (poco claro para mí) fechado en 1255, en el cual detta se glosa con 'debtor', así como otro de 1265 donde el mismo sustantivo se glosa con 'debt to be collected, account receivable'.

Como ya observó Cuervo (1909: 288), la forma septentrional que corresponde al toscano detta es ditta (o dita, con reducción de la consonante geminada).

\footnotetext{
${ }^{7}$ Taula fue el nombre del banco municipal de Barcelona.
} 
En vez de buona/cattiva detta, por ejemplo, se decía bona/cativa dita en Venecia, y formas similares se usaban en Milán, Turín y Génova. A Cuervo le causaba problema que estas formas septentrionales por razones de fonología diacrónica no pueden relacionarse con DEBITA, sino solo con DICTA. Ahora que sabemos que el término detta tiene que relacionarse con DICTA por lo menos en el significado 'giro bancario', el problema de Cuervo se disuelve en la nada. Pero ¿quiere eso decir que la fuente inmediata del término español fue una variedad septentrional del italiano, a lo mejor el genovés, cuyos banqueros tanta importancia tuvieron en la península ibérica? Sería una conclusión precipitada, porque el intermediario entre la palabra italiana y la española fue el catalán.

\section{EL TÉRMINO DITA EN CATALÁN}

Barcelona, por su ubicación geográfica y su vocación mercantil, mantuvo durante toda la Edad Media un estrecho contacto con Italia, el centro del comercio mediterráneo y de las innovaciones en materia de técnica comercial. No sorprende, por eso, que encontremos allí huellas de nuestro término bancario con casi dos siglos de anterioridad respecto de Castilla. Y esas primeras huellas catalanas encajan todavía perfectamente en el uso bancario que acabamos de describir en la sección anterior sobre la base de los trabajos de Federigo Melis. Barcelona, de hecho, fue una precursora en materia bancaria: la ya mencionada Taula de Canvi, inaugurada en 1401, fue el primer banco municipal, anterior incluso al Banco di San Giorgio de Génova, fundado en $1409^{8}$. Pero ya antes de la fundación del banco municipal existían banqueros privados que practicaban el giro entre cuentas para sus clientes. Los libros de uno de esos bancos privados, fechables en el último tercio del siglo XIV, han sido estudiados por Conde y Delgado de Molina (1988). García (1964: 81) ya había hallado "pruebas documentales abundantes en el siglo XIV" de la operación del giro bancario, que en las fuentes se denomina dita. He dicho en la sección anterior que el giro tenía lugar en una reunión de las tres partes involucradas, el banquero y los dos clientes que querían efectuarlo. Esta constelación fue efectivamente la original, pero con el tiempo, para ganar en flexibilidad, se desarrollaron formas más complejas en las cuales intervenían órdenes escritas, sea una especie de cheques sea letras de cambio. Melis (1972: 103) ya encontró tales operaciones en Italia en la segunda mitad del siglo XIV, época en la cual también se documentan en Barcelona (García, 1964: 81; Conde y Delgado de Molina, 1988: 130; Hurtado, 2005: 367-372). Así lo prueba explícitamente el asiento siguiente de los libros estudiados por Conde:

\footnotetext{
${ }^{8}$ Sobre la Taula de cambis de Valencia, posterior también a la de Barcelona, v. Lapeyre (1982).
} 
Deven en Bernat Marquet e.n Gabriel Ros, que dixem per ells a.n Gabriel Ros, fo feta la dita per letra que.n aguem d'en Bernat Marquet he ab volentat d'en Romeu de Busquets ... 64.18.11 (25 de febrero de 1374; Conde y Delagado de Molina, 1988: 131).

Los ejemplos más antiguos de dita García (1964: 82) los encuentra en forma latinizada: "si emptor promisserit venditori facere dictam" (1342), "fecisti mihi cambium seu ditam apud Barchinonam" (1344). Pero ya poco después (1348) aparecen también ejemplos en catalán: "E si lo comprador haurà promès al venedor de fer-li dita del preu en taula, que en tal cars li sia tengut de fer-li la dita en taula a pagar al terme o térmens que serà emprés entre ells" (Furs de Valencia, ed. de 1547, XIII, VI, I).

El libro de Usher (1943: 554-556) contiene un glosario catalán-inglés, en el cual también hay una muy nutrida entrada dedicada a dita. Todos los ejemplos representan el uso técnico de la palabra, del cual Usher distingue dos acepciones:

1. An entry on the books of the bank to the credit of a designated party, whether arising out of a deposit or a transfer on the books; an entry, a credit, a transfer of credit. Cf. the modern partida (Catalan and Spanish). In these documents partida is used in this meaning after the beginning of the sixteenth century (1527), and dita drops out of use.

2. A credit entry on the books of a bank; representing either an authorized overdraft or a loan on a note or bill of exchange. The establishment of this meaning rests primarily upon the accounts of $\mathrm{P}$. des Caus and A. d'Olivella and the accounts of the Bank of Deposit.

De los ejemplos que proporciona Usher, será suficiente citar el más antiguo y el más reciente concerniente a la acepción (1):

Item quens dixem per en Lorent luquer a xxvii Maig en cxxiii carta ... 1 lliures. [Struck out in the text with the following marginal note: Aquesta dita no la faeren per que la cancela aci e en lo compte de en Lorent liques e en manual] ${ }^{-}$Also we credit them with fifty pounds on behalf of Lorent Luquer on the 27 th of May, at page $123 \ldots 50 \mathrm{ll}$. This transfer shall not be made, as it is cancelled here and in the account of Lorent Luquer and in the Journal (AMB, Libro Mayor de la Taula, 1403-1404, fol. 25v).

... fan dites o partides ab polices signadas de lur ma ${ }^{-}$make credit transfers by means of checks signed in their own hand (AMB, Reg. d'ordinacions, 1519-1530, fol. 179v [Oct. 29, 1527]).

Como en español, este uso de dita es hoy obsoleto en catalán. El Diccionari de la llengua catalana del Institut d'Estudis Catalans (1995) lo define así: "ANT. Ordre verbal o escrita d'una operació bancària". Los únicos usos vivos son 'allò que hom diu' y 'allò que hom ofereix de pagar en una venda, per un lloguer, per un arrendament, etc.'. Cabe preguntarse ahora qué relación 
existe entre el catalán y el italiano 9 . En cuanto al significado 'giro bancario', no creo que el cambio de $e$ a $i$, es decir de detta a dita, sea necesariamente indicio de un origen septentrional. La equivalencia detta $=$ dita se podía deducir también de otras equivalencias, por ejemplo entre las locuciones a detta di $X$ y $a$ dita de $X$, esta última documentada para el catalán en Alcover y Moll (2005 [1951-1962]), s. v. dita. Por contra, el significado 'oferta' que sirvió a Corominas para argumentar a favor de un origen catalán, es completamente independiente de nuestro dita bancario; se trata más bien de otra extensión semántica natural del sentido 'allò que hom diu', que a su vez viene directamente del latino DICTA. Este uso, que el español desconoce, sí existía también en el occitano antiguo (v. FEW III 68a: "Apr. dicha 'paroles, propos; offre (dans une enchère)". En cuanto a los significados 'deuda' y 'deudor', que son los que encontramos en los primeros documentos españoles del siglo XVI, me parece indudable que tienen que ver con extensiones metonímicas del uso bancario, pero no podemos decir con certeza si son evoluciones independientes en italiano y en catalán o si estos usos también constituyen calcos. En vista de la dirección general de las corrientes de difusión de ideas y palabras entre Italia y Cataluña, muy especialmente en el ámbito comercial, la hipótesis del calco semántico aparece naturalmente favorecida, a pesar del adelanto de los barceloneses en la constitución de su banco municipal.

Terminaré esta sección sobre el catalán con la descripción del uso de nuestro término en las cartas comerciales de un mercader valenciano de finales del siglo XVI (Iborra Lerma y Vila López, 2013), que puede pasar por representativo del lenguaje comercial de su tiempo. Este mercader alternaba el catalán con el español según el destinatario a quien se dirigía. En las cartas escritas en catalán, aparecen con frecuencia los significados 'deuda' y 'deudor', este último sobre todo en la expresión bona dita 'mercader de gran crédito':

Veix me avisa com les dites que se me dehuen ay per a octubre que ve són de òmens molt abonats y bons, especialment la dita que's de Micalàngel Làmbies y que por lo tant nos determina de trocar-les, puix lo temps és curt i la coginilla va molt cara. A d'axiò dich a V.m. que si los deutors són bons [...] i les dites li parexien molt bones, no les toque i no les troque a ninguna cosa, i quant no ni paregués en tals, farà de trocar-les a coginilla que sia bona i que no li coste més de 52 ducats arrova [...] (15-7-1590, p. 33).

Procurareu de haver bones dites i donar-les a cambi per a d'asi (15-7-1590, p. 34).

En algún que otro ejemplo parece que podríamos incluso entrever el significado 'partida', que Usher decía que ya estaba saliendo del uso a inicios del

\footnotetext{
${ }^{9}$ Dita falta en el apartado dedicado a los derivados de dir en el DECat (III 143b) de Coromines.
} 
siglo. Pero la verdad es que 'deuda' es también una lectura aceptable, aunque a lo mejor poco elegante, en el siguiente ejemplo. Este caso muestra con qué facilidad se podía pasar de un significado a otro en determinados contextos:

Reste avisat que si hi à lloch de fer tollines del duch, barato les dites que aí se me dehuen" (8-6-1591, p. 51).

Las cartas escritas en español son enteramente parecidas:

el dicho Grau soplica a V.m. le haga mercé venda sus piesas de contado o fiados a huna o dos ditas que sean muy buenas (8-11-1590, p. 43).

Enviarme ha la cuenta de dichas tres piesas i también de las demás y si V.m. halla ocasión de huna buena cochinilla, V.m. trueque las ditas de las tres piesas que m'avisa tiene vendidas, fiadas ocho meses a Joan Abat a 19 reales vara (3-1-1592, p. 76).

Nuestro mercader también utiliza la expresión estar de las ditas, que corresponde al italiano star del credere, es decir, garantizar (el socio) el pago de las mercancías vendidas a plazo por él:

V.m. mande procurar que se venda esa seda de contado al presio que mejor pudiere y quando no, V.m. me la fie para la feria de junio de Medina del Campo al más presio que pudiere, con que si querrá estarme de las ditas, tomarse a V.m. doble responsión, i quando no, V.m. las venda a los [sic] mejores ditas que pudiere (8-1-1594, p. 163).

Otra vez tenemos aquí un contexto en el cual resulta casi imposible decidir si dita significa 'deuda' o 'deudor'.

\section{CONCLUSIÓN}

Concluyendo, pienso que a la luz de los materiales y argumentos aducidos el origen y las complejas andanzas del término económico dita, cuya etimología el DRAE presenta como "discutida", pueden considerarse como esclarecidos en sus grandes líneas. Tenemos que partir de un significado que ya no es usual en ninguna lengua románica, es decir, 'giro bancario' y, por una extensión metonímica natural, 'partida de una cuenta' (materialización del giro). Este uso bancario original fue, sin lugar a dudas, una aplicación a un contexto específico del significado general 'enunciado' heredado del sustantivo latino DICTA, neutro plural de Dictum. Se refería inicialmente al enunciado con el cual el banquero selló la operación. Aunque la operación permitía muy pronto el uso de órdenes escritas, el término técnico original siguió usándose por inercia, como bien vio Conde y Delgado de Molina (1988: 134): 
Excepto en una determinada operación en que la cantidad a girar se le "dice" a la Banca (la transferencia de créditos desde una banca a otra), el sujeto gramatical del verbo decir es siempre la Banca: que dixem per ell a o que li dixem per. Ciertamente, en un primer estadio tal vez el dir puede hacer referencia a una presencia física, pero en estos momentos el lenguaje se ha fosilizado y separado de su significado primitivo. Y no es el único caso: aun hoy día se habla de "letras de cambio" cuando a través de ellas no se realiza ningún cambio.

Las nuevas circunstancias habrán contribuido a oscurecer la motivación del término, alejándolo de la familia de dire/dir. En español, por supuesto, el parentesco tenía que resultar opaco desde el inicio, ya que el participio de decir era dicho y no dito. El oscurecimiento de la motivación original puede también haber facilitado las extensiones metonímicas posteriores a 'deuda' y 'deudor'. El paso de 'partida' a 'deuda' es bastante lógico, ya que las deudas y los créditos se materializaban como partidas en los libros de un banquero. El último paso, de 'deuda' a 'deudor', tampoco tiene nada de extraño. De hecho, los mercaderes italianos de entonces solían también decir metonímicamente de un colega de gran crédito que era una "buona lettera", es decir, que solía honorar las letras de cambio que se libraban sobre él:

Giovanni [...] priega un sansale che gli ritruovi una buona lettera (ch'è un trattante sicuro), al quale possa dare questa moneta a cambio (Marco Palescandolo, finales del siglo XVI, en Cassandro, 1962: 119).

Quel sopra più, che mi vien dato, per esser' io ricco, e stimato buona lettera (Bernardo Giustiniano, Breve trattato..., Genova, Pavoni, 1619, p. 181).

La extensión de 'deuda' a 'deudor', por ende, probablemente también tuvo lugar en italiano, aunque no tenemos ejemplos anteriores al siglo XVI, el mismo periodo cuando este uso se documenta en catalán y en español. El español, de todos modos, fue una lengua puramente receptora. Con toda probabilidad, el español dita es un préstamo del catalán en ambas acepciones, 'deuda' y 'deudor'. La documentación disponible no nos permite asegurar si el español también tomó prestado los sentidos más estrechamente técnicos 'giro bancario' y 'partida'.

Esta modesta nota etimológica quizá pueda reclamar también cierto interés en un contexto más amplio, come sugiere un anónimo evaluador. En el debate sobre el origen de la moneda, algunos economistas se han servido de la etimología como ciencia auxiliar para penetrar las tinieblas de los tiempos prehistóricos. Así, por ejemplo, Ingham (2004: 90), en un pasaje citado y avalado también por Graeber (2011) en el capítulo 3 de su libro dedicado al supuesto origen sacrificial de la moneda, escribe:

In all Indo-European languages, words for 'debt' are synonymous with those for 'sin' or 'guilt', illustrating the links between religion, payment and the mediation of the sacred and profane realms by 'money'. For example, there 
is a connection between money (German Geld), indemnity or sacrifice (Old English Geild $[\text { sic }]^{10}$ ), tax (Gothic Gild) and, of course, guilt (Hudson, 2004).

No es este el lugar para entrar en la relación exacta, desde el punto de vista de la cronología de las extensiones semánticas, de las palabras germánicas mencionadas en esta cita (véase, al respecto, Benveniste, 1969: 70-74). Lo que está a la vista es que la aserción es demasiado abarcadora. Incluso si fuera correcta para las lenguas germánicas, no cabe duda de que no se aplica a las lenguas indoeuropeas en su conjunto. Las palabras más comunes para decir 'deuda' en las lenguas románicas (esp. deuda, cat. deute, fr. dette, etc.), por ejemplo, para no ir más lejos, remontan al latino DEBITUM o su plural tardo-latino DEBITA, a su vez participios sustantivados de DEBERE 'deber (dinero)' (significan literalmente 'lo debido'), y DEBERE representa una forma contraída de *DE-HABERE, cuyo probable significado fue 'retener' según Walde (1938). No parecen entrar aquí ni culpas ni pecados, como tampoco en el caso de nuestro dita que, como he argumentado, fue calco del italiano detta, que a su vez tiene como étimo latino DICTA 'lo dicho, enunciado'. El cambio semántico sorprendente de 'lo dicho, enunciado' a 'deuda' se debió a la peculiar función que tenía el 'enunciado' del banquero en el acto del giro bancario. Estos dos ejemplos muestran que las vías que conducen al significado 'deuda' son múltiples y a veces bastante enrevesadas. Podría merecer la pena que alguien dedicara un día a esta temática un amplio estudio onomasiológico, de interés no solo para la lingüística histórica sino también, aparentemente, para economistas.

La Figura 1 sintetiza la suerte que, según nuestra reconstrucción, el sustantivo latino DICTA corrió a través de los siglos en italiano, occitano, catalán y español. El significado original de DICTA será dado como 'enunciado' por pura conveniencia. Omito, para no complicar las cosas, la acepción 'fortuna' de DICTA.

${ }^{10}$ La forma correcta es Gield. Muy significativamente, los más de 100 escritos que Google proporciona cuando se busca "Old English Geild" fueron escritos por economistas, que habrán tomado como fuente la cita de Ingham... 


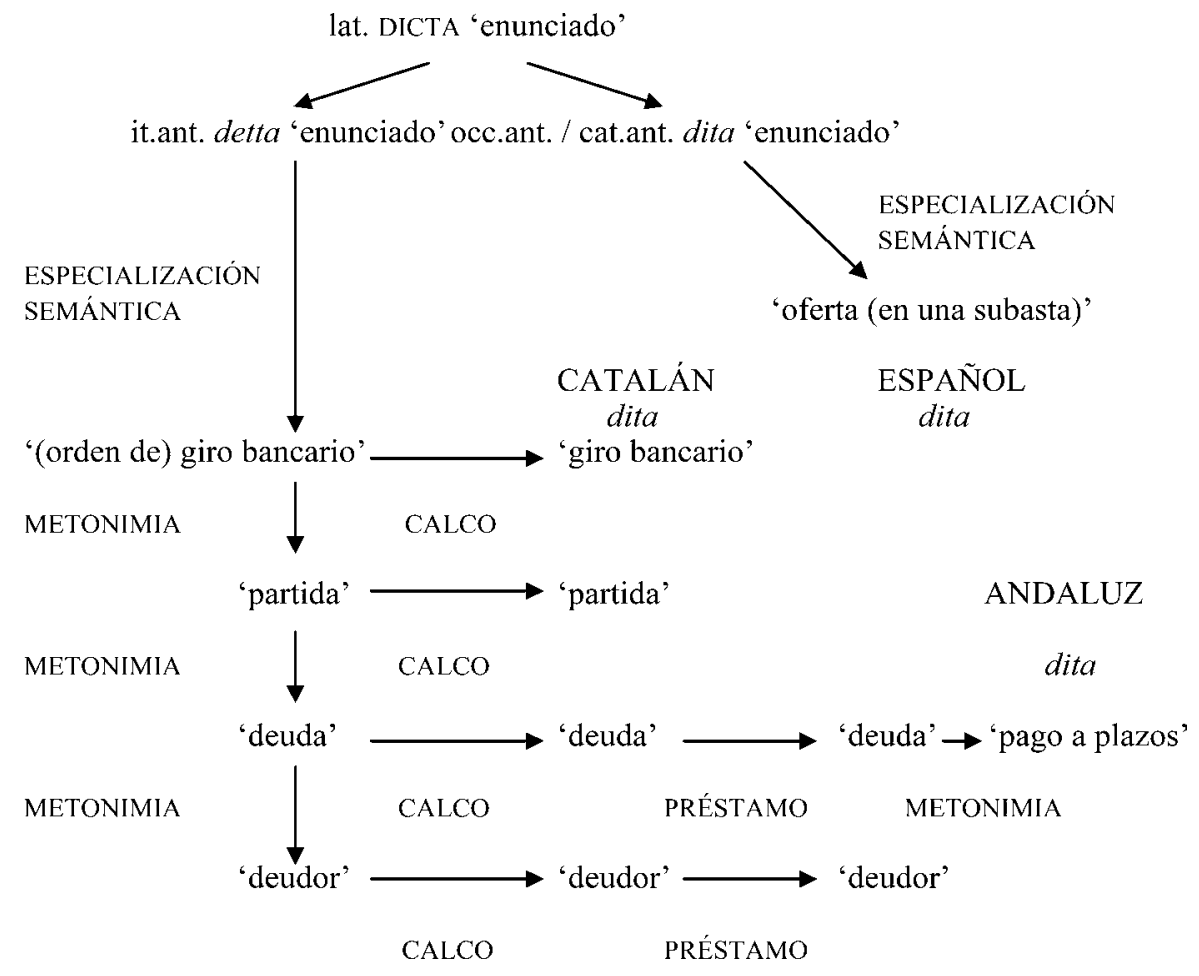

FiguRA 1.- Suerte de DICTA en cuatro lenguas románicas.

\section{BIBLIOGRAFÍA}

Accademia della Crusca (1612): Vocabolario, Venezia, Alberti, <http://www.lessicografia.it/>. Alcalá Venceslada, Antonio (1951): Vocabulario andaluz, Madrid, Aguirre.

Alcover, Antoni M. ${ }^{\mathrm{a}}$ y Francesc de B. Moll (2005 [1951-1962]): Diccionari català-valencià-balear, Palma de Mallorca, Miramar.

Alvar Ezquerra, Manuel (2000): Tesoro Léxico de las Hablas Andaluzas, Madrid, Arco/Libros. Asociación de Academias de la Lengua Española (2010): Diccionario de americanismos, Madrid, Santillana. Beltrán de Heredia, Vicente (ed.) (1935): Comentarios a la Secunda secundae de Santo Tomás, vol. IV, Salamanca, Biblioteca de Teólogos Españoles.

Benveniste, Émile (1969): Le vocabulaire des institutions indo-européennes. Vol. 1: Économie, parenté, société, Paris, Les Éditions de Minuit.

Calero López de Ayala, José L. (1987): Léxico alcarreño conquense: aproximación al estudio etnolingüístico de la comarca, Cuenca, Excelentísima Diputación Provincial de Cuenca.

Carande, Ramón (1978): Otros siete estudios de historia de España, Barcelona, Ariel.

Cassandro, Giovanni (1962): Un trattato inedito e la dottrina dei cambi nel Cinquecento, Napoli, ESI.

Company, Concepción y Chantal Melis (2002): Léxico Histórico del Español de México, México, UNAM.

Conde y Delgado de Molina, Rafael (1988): "Las actividades y operaciones de la banca barcelonesa trecentista de Pere Descaus y Andreu d'Olivella", Revista Española de Financiación y Contabilidad, XVII (55), pp. 115-169. 
CORDE = Real Academia Española: Banco de datos (CORDE) [en línea]. Corpus diacrónico del español, <http://www.rae.es>.

Cuervo, Rufino José (1909): "IV. Dita", Bulletin Hispanique, 11, pp. 287-294.

DCECH = Juan Corominas y José Antonio Pascual (1980-1991): Diccionario crítico etimológico castellano e hispánico, Madrid, Gredos.

DEA = Olimpia Andrés, Manuel Seco y Gabino Ramos (1999): Diccionario del Español Actual, Madrid, Santillana.

DECat $=$ Joan Coromines (1982): Diccionari etimològic i complementari de la llengua catalana Barcelona, Curial.

DELI = Manlio Cortelazzo y Paolo Zolli (1979-1988): Dizionario etimologico della lingua italiana, Bologna, Zanichelli.

DRAE = Real Academia Española (2014-): Diccionario de la lengua española, $<\mathrm{http}: / /$ dle.rae.es $>$.

Edler, Florence (1934): Glossary of Medieval Terms of Business. Italian series 1200-1600, Cambridge, The Mediaeval Academy of America.

FEW = Walther von Wartburg (1928-2002): Französisches Etymologisches Wörterbuch, Bâle, Zbinden. García, Arcadio (1964): "El contrato de «dita» y la letra de cambio", Ausa, 49, pp. 81-87.

$G D L I=$ Salvatore Battaglia (ed.) (1961-2002): Grande dizionario della lingua italiana, Torino, UTET.

Goris, Jan Albert (1925) : Étude sur les colonies marchandes méridionales (Portugais, Espagnols, Italiens) à Anvers de 1488 à 1567, Louvain, Librairie Universitaire.

Graeber, David (2011): Debt. The first 5000 Years, New York, Melville.

Hudson, Michael (2004): "The archaeology of money: Debt vs. barter theories of money", en Randall L. Wray (ed.), Credit and State Theories of Money, Cheltenham, Elgar, pp. 99-127.

Hurtado, Víctor (2005): Llibre de deutes, trameses i rebudes de Jaume de Mitjavila i companyia 13451370. Edició, estudi comptable i econòmic, Barcelona, Consell Superior d'Investigacions Científiques.

Iborra Lerma, José Manuel y Margarita Vila López (eds.) (2013): Cartes comercials i lletres de canvi de Francés Crespo, mercader valencià (1585-1601), València, Universitat de València.

Ingham, Geoffrey (2004): The Nature of Money, Cambridge, Polity Press.

Institut d'Estudis Catalans (1995): Diccionari de la llengua catalana, Barcelona, Edicions 3 i 4.

Lapeyre, Henri (1982): La Taula de cambis (en la vida económica de Valencia a mediados del reinado de Felipe II), Valencia, Del Cenia al Segura.

$L E I=$ Max Pfister (1984-): Lessico etimologico italiano, Wiesbaden, Reichert.

López Blanquet, Marina (1992): Uruguayismos, Montevideo, Monteverde.

Melis, Federigo (1962): Aspetti della vita economica medievale, Siena/Firenze, Monte dei Paschi di Siena/Olschki.

Melis, Federigo (1972): Documenti per la storia economica dei secoli XIII-XIV, Firenze, Olschki.

Morínigo, Marcos A. (1985): Diccionario de americanismos, Barcelona, Muchnik.

$N T L E=$ Lidio Nieto Jiménez y Manuel Alvar Ezquerra (2007): Nuevo Tesoro Lexicográfico del Español (s. XIV-1726), Madrid, Arco/Libros.

Oudin, César (1616): Tesoro de las dos lenguas francesa y española, Paris, Orry.

Real Academia Española (2002 [1732]): Diccionario de Autoridades, Madrid, Gredos.

Terlingen, Johannes Hermanus (1953): Los italianismos en español desde la formación del idioma hasta principios del siglo XVII, Amsterdam, Noord-Hollandsche Uitgevers Maatschappij.

Trolli, Domizia (1998): "Postilla a ditta (di banco)", Lingua Nostra, 59, pp. 20-21.

Usher, Abbott P. (1943): The Early History of Deposit Banking in Mediterranean Europe, Cambridge, Harvard University Press.

Walde, Alois (1938): Lateinisches etymologisches Wörterbuch, vol. 3, Johann Baptist Hofmann (ed.), Heidelberg, Winter.

Fecha de recepción: 16 de febrero de 2017

Fecha de aceptación: 27 de marzo de 2017 